\title{
Associated Movements of the Little Finger during the Precision Grip with the Thumb and Index Finger
}

\author{
Mitsuo Nakamura ${ }^{1}$, Mariko Nakamura ${ }^{1}$, Yuji Sawada ${ }^{2}$ \\ ${ }^{1}$ Department of Occupational Therapy, Graduate School of Health Sciences, Sapporo Medical University \\ ${ }^{2}$ Department of Occupational Therapy, School of Health Sciences, Nagoya University
}

\begin{abstract}
We studied the relationship between the coordinated precision grip force of the thumb and index finger and the positions and associated movements of the little finger. Subjects $(n=10)$ pinched the pinch-meter and followed the target wave, which was made by a triangular wave and two horizontal waves. When following the target wave (TW) with precision grip, subjects preformed two tasks: changing the little finger positions to neutral, flexion and extension (Task-1) and performing flexed and extended movements (Task-2). The errors between TW and the following wave resulting from precision grip with the thumb and index finger were recorded. We found that the change of the little finger position and joint movements influenced the coordinated precision grip force. Furthermore, we found a relationship between the plus or minus directions of error, and the direction of the little finger's joint movements and little finger positions. This study indicates that the little finger position influences coordinated precision grip force, but showed no involvement between the thumb and the index finger.
\end{abstract}

Keywords: power coordination, little finger, precision grip

(Asian J Occup Ther 11: 1-8, 2016)

\section{Introduction}

In our daily living, we can grasp and operate manifold objects appropriately. For this to be accomplished, we coordinate both a complex system of sensory-receptors and motor skills with the object's properties. Finding normal finger functions and coordinating fingers are necessary for occupational therapists when providing therapy for the loss of finger functions in orthopedic and cerebral vascular disease patients. There are two prehension synergies used when holding an object in static tasks by individual digits. The first is to change the hand shape and configuration according to the object's shape, weight, friction and center of gravity, and the second is to grasp and operate the objects. According to Napier (1956), even though we have grasped the same object, prehensile activities can be classified into the two categories of 'power grip' and 'precision grip'. Power grip is

Received: 20 January 2015, Accepted: 10 December 2015

Corresponding to: Mitsuo Nakamura, Department of Occupational

Therapy, Graduate School of Health Sciences, Sapporo Medical University, west 17 south 1, chuo-ku Sapporo, 060-8556, Japan

e-mail: micchan@sapmed.ac.jp

C2016 Japanese Association of Occupational Therapists used to fix objects in the hand with the palm. Precision grip is used for the purpose of fine control of prehensile forces on a manipulated object, which is usually held by the tips of the fingers and opposing thumb. Kamakura (1978) found that the shape of the hand while grasping an object was decided by the purpose and operation of the grasp; furthermore, the contact positions between the object and hand when grasping a large number of objects were classified. This classification indicated that the contact positions varied by individual. However, Kamakura also revealed that the characteristic contact positions were derived from the physical properties of objects according to the intent of the grasp and operation. The second synergy is to coordinate the grip and pinch power to accomplish the smooth grasp and operation of the object. Previously, power coordination was measured using the precision grip between thumb and index fingers, which is the minimum unit of grasping. According to Johansson \& Westling (1984), an appropriate amount of grip and pinch power was provided when people grasped objects depending on the object's material and weight, so that the object would not to slide out. They found that the precision grip power when holding an object in space consisted of two elements: minimum pinch power requirements, and redundant power (safety 
margin). Furthermore, Nakamura (1993) reported that subjects provided $5 \sim 7 \%$ of maximum precision grip force during perturbation when vertical load force was applied suddenly to the grip apparatus. Goto (2003) found that fixing the wrist joint influenced the finger joint's motion during precision grip between the thumb and index finger. In terms of operating environment, Nakajima (2012) indicated that an obstacle's position influenced the trajectory and velocity of precision grip. Yamashita (2005) found that the thumb and index finger each have a functional role during this precision grip. When EMG amplitude was measured using graded increases of precision grip force, the thumb took on a 'fixed' role (FR) and index finger had the 'role of gradual power coordination' (GPC) showing no change in the thumb's precision amplitude but a gradual change in the index finger. Coordinated precision grip force and the functional role between the thumb and index finger has been reported. However, many kinds of object-dependent tasks are carried out in daily life that require skilled manipulations with not only a thumb-index finger pinch but also whole-handed manipulations involving all five digits. Recent studies on whole-hand grasping have shown that the force of individual digits is related to the direction of rotational torque in the rotational equilibrium for hand-held objects (Zatsiorsky et al., 2003). According to Taguchi (2002), when rotating a circular cylinder, it was found that the little finger used a highly coordinated movement. The results of chaos analysis of the trajectory of the five fingers showed that the index and middle fingers were highly chaotic, while the thumb and little finger showed little chaos (8). Hence, each finger assumed a functional role during grasping and operation, the middle, ring and little fingers taking on a functional role during a precision grip between the thumb and index finger. This study investigated the little finger's functional role during a precision grip between the thumb and index finger. If the little finger is not directly engaged in prehension, but is involved in the coordinated precision grip force between the thumb and index finger, it may be necessary to consider that the functional unit is not each finger but the whole hand, with all fingers influencing one another.

\section{Method}

\section{Participants}

Ten right-handed male and female volunteers (average age $25.8 \pm 4.80$ years old) were selected as subjects for this study. None had previous histories of neuropathies or trauma in the upper limbs. All volunteers gave informed consent according to the procedures approved by the ethics committee of Sapporo Medical
University.

\section{Apparatus and experimental setup}

A pinch meter (PM) was used (Fig. 1) which was horseshoe shaped and consisted of two parallel stainless bars and a strain gauge, and which was attached to a baseboard. Amplified electric signal waves measuring grip between the thumb and index finger were produced on the PM, and shown on the oscilloscope (DL1640, Yokogawa, Japan). The oscilloscope was set at the subjects' eye level, and its display preferences were fixed to a vertical axis of electric voltage $2.0 \mathrm{v} / \mathrm{div}$, and horizontal axis of recording time $500 \mathrm{~ms} / \mathrm{div}$. Additionally, the target wave (TW) was projected onto the oscilloscope. The TW was made by a functional generator (FG-272, KENWOOD, Japan). The TW consisted of a triangular wave and two horizontal lines. The triangular wave was $0.2 \mathrm{~Hz}$ and the amplitude was set at $10 \%$ of maximum voluntary contraction (MVC). The two horizontal lines were drawn in both the upper and lower regions of the triangular wave in the oscilloscope. We temporarily recorded the TW and following wave, which were made from the PM in the oscilloscope by a data-recorder (RD-135T, TEAC, Japan). The recorded waves were analog-digital converted with a sampling rate of 1,000 $\mathrm{Hz}$ by an A/D converter (AND-1400, canopus, Japan). After A/D conversion, the digital wave data were recorded using a PC (PC9821Ne, NEC, Japan) and analyzed with Microsoft Excel 2000 software on a standard EPSON Type-VF computer.

\section{Tasks}

To determine whether the little finger is related to the coordinated precision grip force between the thumb and index finger, subjects were asked to match the target

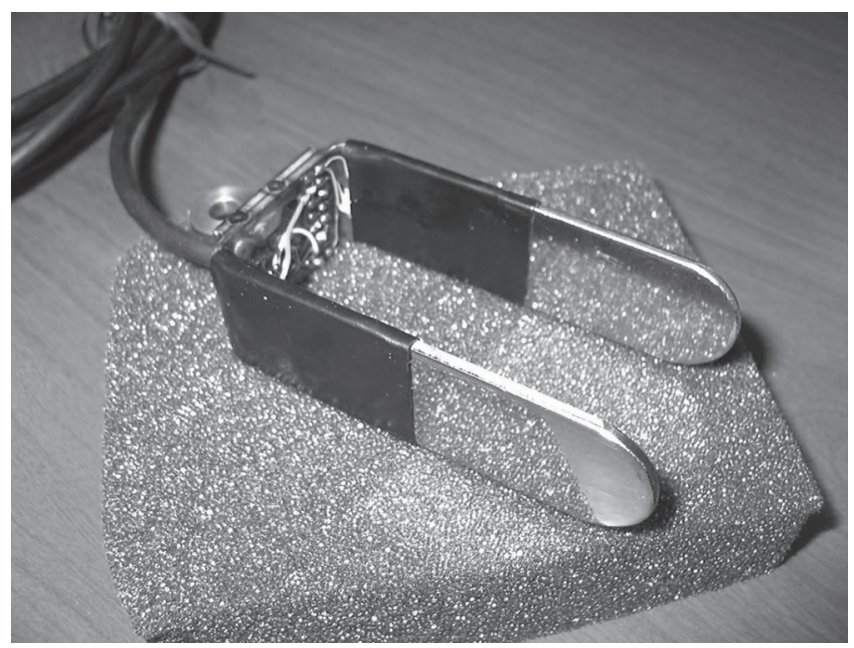

Fig. 1. Pinch Meter (PM). 
wave with the following wave made by precision grip force. In this study, two task variations were set (Task-1, Task-2) to investigate the power coordination between the motion position of the joints' of the little finger and precision grip force.

Task-1

In Task-1, subjects pinched the PM with the thumb and index finger (Fig. 2a). On the oscilloscope display, three lines emerged: a triangular wave and two fixed horizontal lines in the upper and lower axis. When the subjects held the PM with minimum pinch power, the following wave line descended to the lower line. When the triangular wave descended to match the lower horizontal line, subjects estimated the timing of when to start the task, and the subjects would start following the TW. Upon applying pressure to the PM, the following wave line ascended. Subjects would have to follow the ascending TW. After reaching the maximum amplitude of the TW, subjects would have to maintain the upper horizontal line position. After 1 up and down cycle of the triangular wave, subjects estimated the timing of when to start the descent when TW was at the upper horizontal line. Subjects then followed the TW from the upper horizontal line to the lower horizontal line. In Task-1, 3 positions of the little finger were purposely set (neutral, flexion and extension of MP, PIP and DIP joints). Trial 1 was completed after two cycles. Subjects did 3 trials with each of the little finger positions $(2 \times 3$ $\times 3=18$ trials $)$.

Task-2

Task-2 investigated the influence of muscle contraction and movements of the joints of the little finger during the coordinated precision grip force between the thumb and index finger (Fig. 2b). The timing to start the following in Task 2 was identical to that of Task 1. At the start of following, the position of little finger remained in a neutral position. Upon applying pressure to the PM, the wave line to be followed was raised, and subjects had to follow it. After reaching the maximum TW amplitude, subjects maintained the position of the upper horizontal line and move the little finger joints while maintaining the position. Upon one ascending-descending cycle after the TW arrived at the upper horizontal line, the subjects flexed the little finger joints from the neutral position to an extended position. With flexion of the little finger, subjects coordinated the precision grip force for one period. Then the subjects flexed their little fingers, closing them back to the neutral position. After remaining in the neutral position for one period, subjects extended the little finger joints from the neutral position, and extended the precision grip for 1 period. In the next period, subjects changed their little fingers' position from extension to neutral, and followed the TW from the upper horizontal line to the bottom line. Subjects repeated the process in 3 trials.

\section{Procedure}

Subjects were given an orientation session before the experiment to become familiar with the experimental apparatus and to ensure that they were able to accomplish the tasks. Before the experiment, subjects washed their hands with soap to normalize their skin, and sat in a chair with the elbow joint rested on an armrest and flexed at $90^{\circ}$. The right forearm was placed in an intermediate position to facilitate a natural grasping position for the hand. The pinch meter was fixed in a horizontal position on vertical beams of the armrest and set in front of the subjects' forearm, and the distal side of the

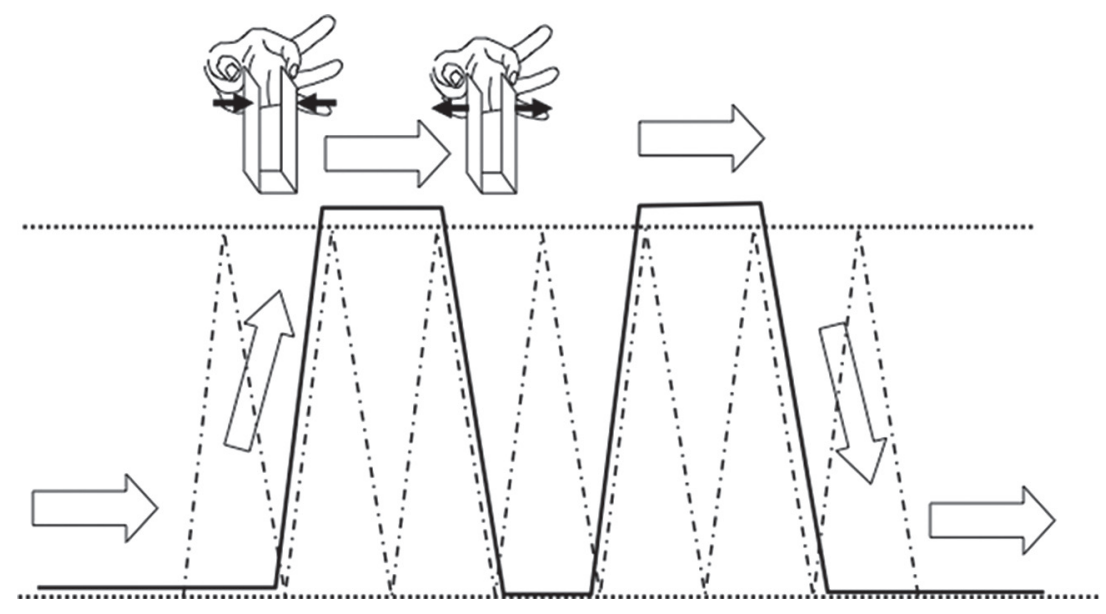

Fig. 2a. Experimental Task-1.

Task-1 was to find whether the change of little finger position (joint angle) influence the power coordination. Dotted line shows upper and lower parameters of waves made from functional generator. Dashed line shows triangular waves displaying period lengths and spacing. Solid line was the target wave (TW) which subjects had to follow. 


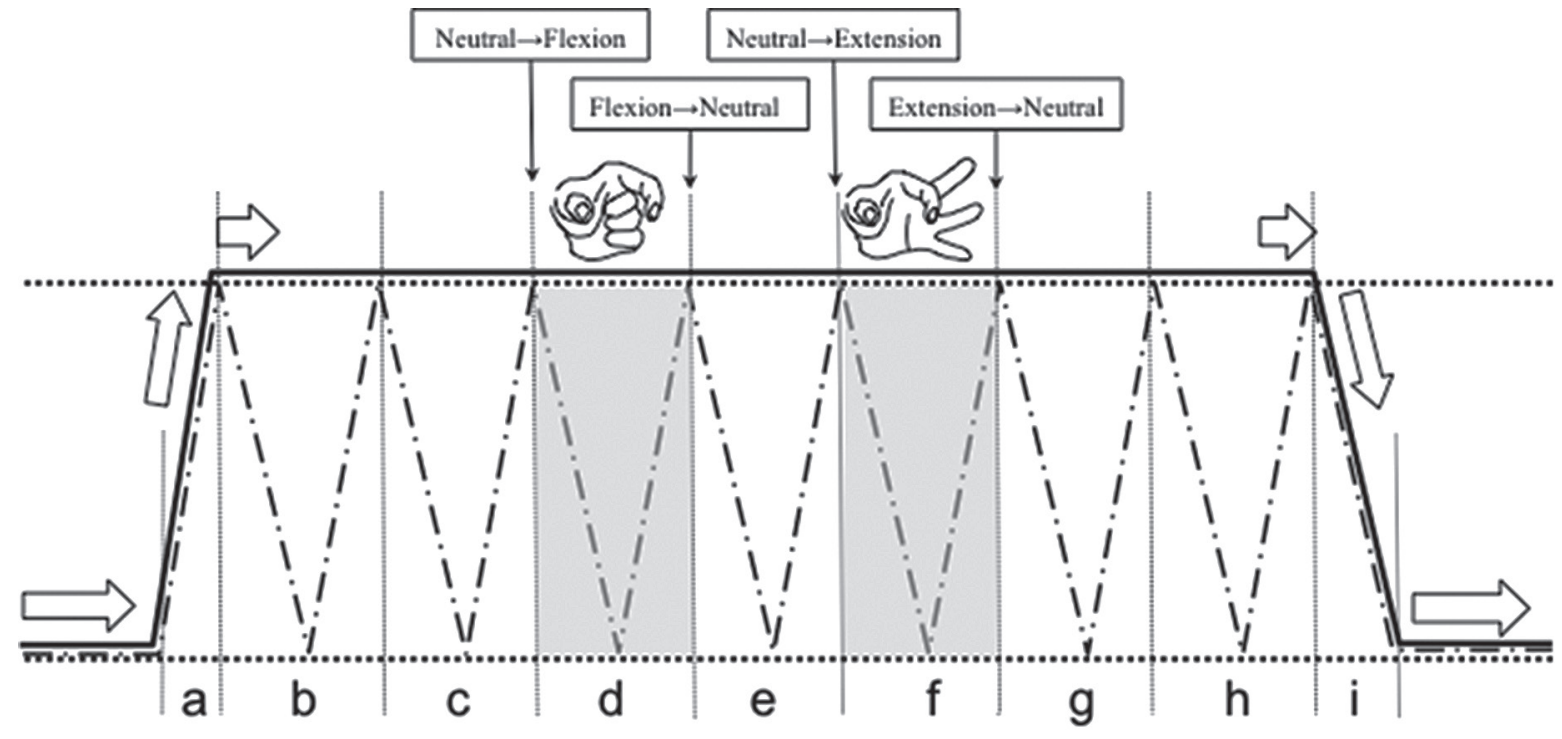

Fig. 2b. Experimental Task-2.

Task-2 was to find the influence for the power coordination of little finger joint movements with precision grip. Dotted line shows upper and lower parameters of waves made from functional generator. Dashed line shows triangular waves displaying period lengths and spacing. Solid line was the target wave (TW) which subjects had to follow. "b", "c" and "h" periods were neutral positions of little finger. "d" period was little finger flexed period and "f" period was little finger extended period. "e" and "g" periods were neutral positions including the rapid time of changing fingers position.

subjects' wrist joint was left unobstructed. The oscilloscope was set in front of the subjects, and the subjects monitored the electric wave on the oscilloscope monitor by holding the pinch meter. After the subjects practiced the tasks to ensure smooth performance, the experiment commenced and subjects performed Task-1. Following a short rest to prevent fatigue, subjects performed Task2. During Task-1, the order of the positions of the little finger was arranged randomly in order to prevent a learning effect.

\section{Data processing and statistics}

Data processing was performed using a datarecorder, and statistics were calculated using SPSS10. All statistical analyses were performed at a significance level of $p<0.05$. Data from two waves (TW and following wave) and data from the two horizontal lines (upper and lower) were collected by the data-recorder, then converted using an $\mathrm{A} / \mathrm{D}$ converter from analog to digital (A/D) with a sampling rate of $1,000 \mathrm{~Hz}$. After $\mathrm{A} / \mathrm{D}$ conversion, the digital wave data were recorded in the PC and analyzed by Microsoft Excel 2000. 90 data entries from Task-1 and 30 data entries from Task-2 were analyzed. The error between TW and following wave were calculated as follows: Raw Error = following wave - TW.

Both plus and minus types of errors emerged, and were computed. Total errors was calculated as follows: Error $=\Sigma \mid$ Raw Error (following wave - TW). If the error was minor, the level of ability of the coordinated precision grip force between the thumb and index finger was high. During data collection, the time the triangular wave took to travel from the bottom to the upper horizontal lines was 1.25 seconds and the time it took to maintain the force at the upper horizontal line was 2.5 seconds. Therefore, the measurement of data per unit of time was calculated. In Task-1, mean errors were calculated for the three periods of 'compression', 'holding' and 'decompression', and then compared using ANOVA for post hoc test. In Task-2, mean errors were calculated for each of the 'compression', 'neutral', 'flexion', 'extension' and 'decompression' periods, and then compared using ANOVA for post hoc test. Testing for significant difference between Task-1 and Task-2 was done using the Tukey-Kramer test performed at a significance level of $\mathrm{p}<0.05$.

\section{Results}

\section{Relationship between little finger position and power coordination}

Fig. 3 shows the errors between TW and following wave at each of the periods: compression, holding and decompression in each little finger position (neutral, flexion, and extension) in Task-1. The errors of the three periods proved to be similar in all little finger positions. The errors of the reduced zone were the largest and those of the holding zone were the lowest of the three periods. 
The error of each period was significantly different by ANOVA ( $p<0.05)$, so the Tukey-Kramer test was used. The error of the continuous holding period was significantly lower than both the compression period and the decompression period $(\mathrm{p}<0.05)$. Compared to the little finger positions, the neutral position of the little finger had the lowest tendency of error of the three little finger positions. Fig. 4 shows the characteristic errors of the holding period in the three little finger positions. The two errors were separated from raw errors. The plus error of the following wave was greater than that of the TW, and the minus error of the following wave was lower than that of the TW. When comparing the plus and minus errors, the plus error was significantly larger than the minus error in the flexed position of the little finger $(p<0.05)$. When comparing the plus and minus rates of the little finger in the flexed position, the plus errors were significantly larger than the minus errors $(p<0.05)$. Furthermore, the minus rate errors of the little finger in the extended position were significantly larger than the errors of little finger in the flexed position $(\mathrm{p}<0.05)$.

\section{Relationship between voluntary movement of little finger and power coordination}

Fig. 5 shows the difference in errors due to changing the little finger positions from neutral to flexion to extension in Task-2. We computed the mean data of each little finger position for the compression and decompression periods. ANOVA tests confirmed that the difference in errors of each little finger position and power coordination over the compression, neutral, flexion, extension, and decompression positions were

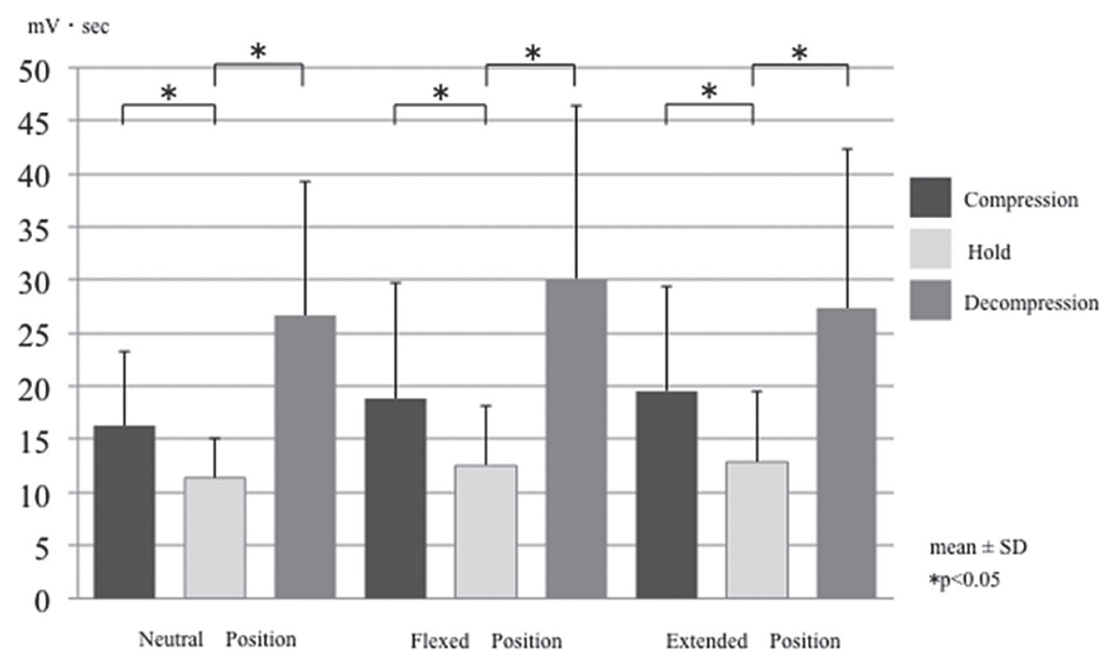

Fig. 3. Error Difference of the Three Little Finger Positions During the Three Conditions (Compression, Hold, and Decompression) in Task-1.

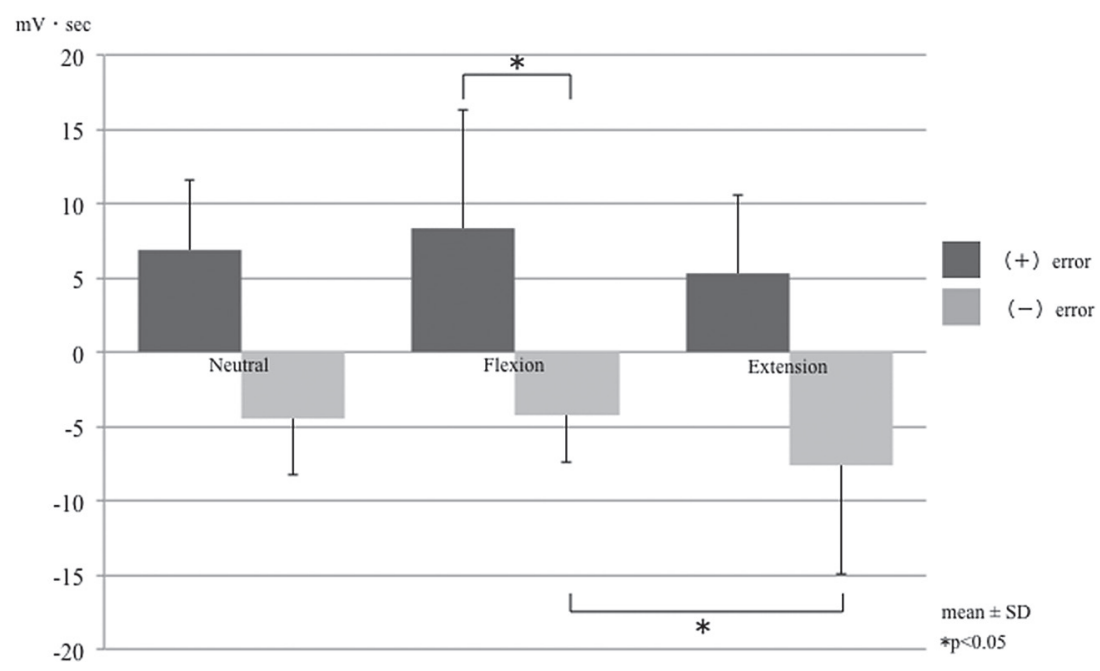

Fig. 4. Differences in Error of the Little Finger Positions During Holding Period (Task-1). 


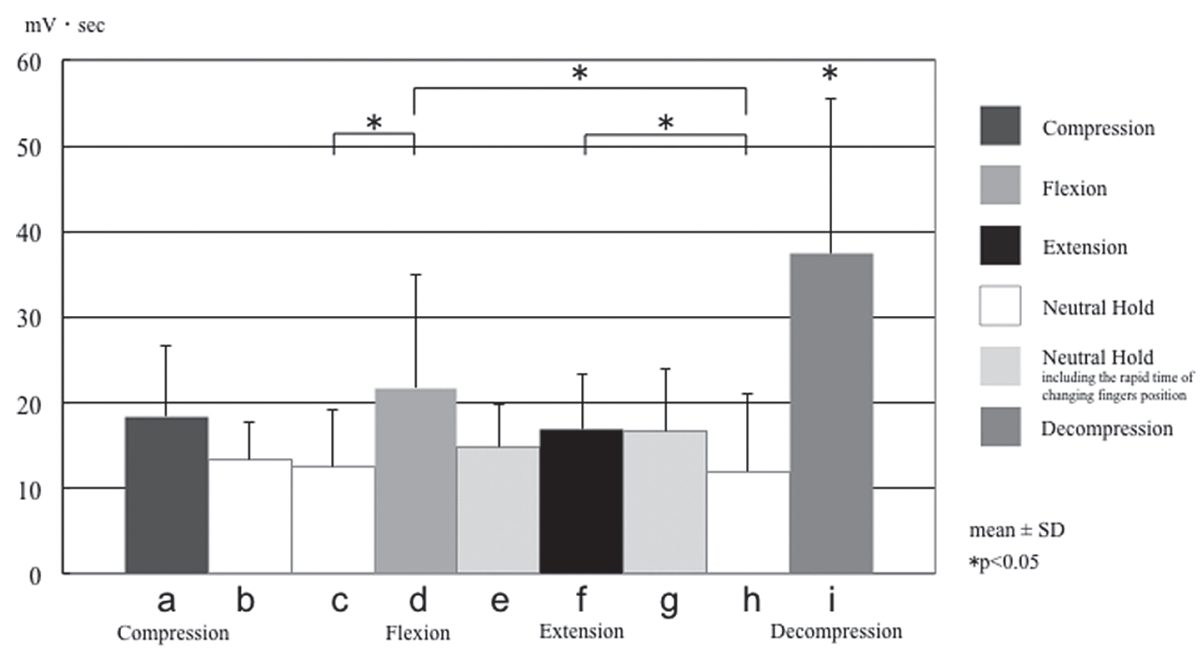

Fig. 5. Difference in Error by Changing Little Finger Positions (Neutral, Flexion, Extension) in the Task-2.

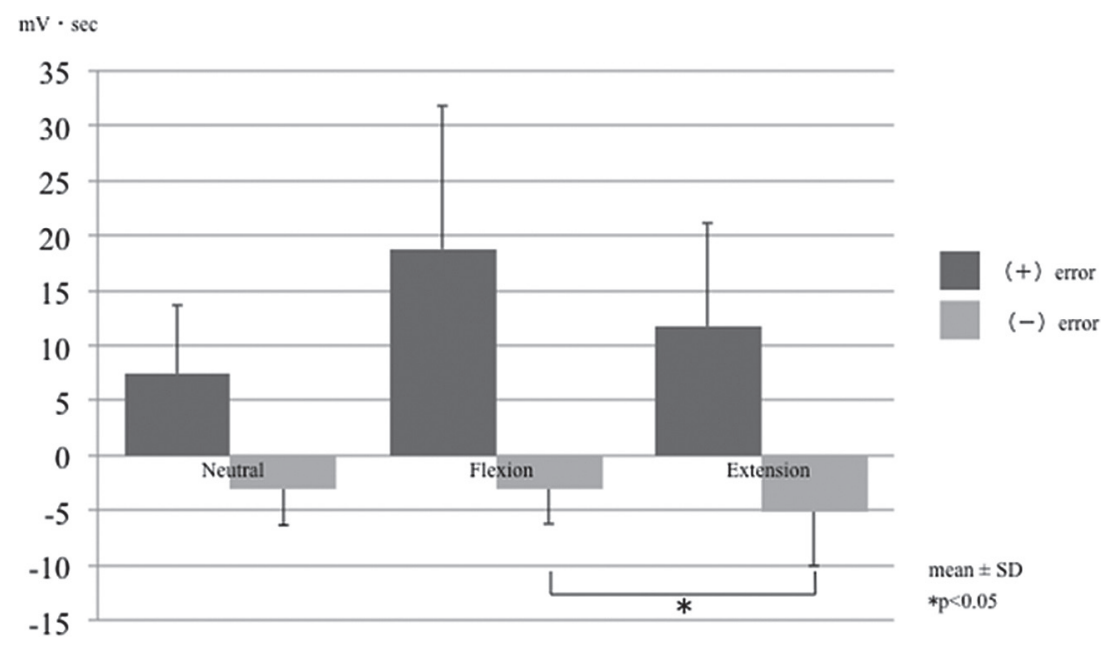

Fig. 6. Difference in Error Among the Three Little Finger Movements (Task-2).

statistically significant $(\mathrm{p}<0.05)$. The results of the Tukey-Kramer test were significant between the periods of neutral position and flexion, and between the periods of the neutral position of the little finger and extension $(\mathrm{p}<0.05)$. Furthermore, the differences in errors among all periods and the decompression period were statistically significant $(\mathrm{p}<0.05)$. Raw errors were separated into plus and minus errors during flexion of the little finger (Fig. 5d), extension of the little finger (Fig. 5f), and neutral position of the little finger (Fig. 5b, c, h). The errors were then compared after separation. Regarding plus errors, the errors of the flexion of the little finger were the largest of the two periods. For minus errors, the errors of the extension of the little finger were the largest, and the difference of errors between the extension of the little finger and the errors of flexion of the little finger was statistically significant $(\mathrm{p}<0.05)$ (Fig. 6).

\section{Discussion}

Task-1 and Task-2 experiments showed that keeping the little finger in a neutral and relaxed position produced the most coordinated force, and that a relationship existed between the plus or minus direction of error, and the direction of the little finger's joint movements and position. Regarding the characteristics of the little finger, the flexor muscles of the little finger were the flexor digitorum superficialis (FDS) and the flexor digitorum profundus (FDP), which were the same for the index finger. In this study, the coordinated precision grip force between the thumb and the index finger was performed 
by using the FDS and the FDP. Yamashita found that the thumb and index finger each have a functional role during precision grip (5). When the little finger was in the flexed position, a plus error occurred which was larger than the other positions of the little finger. This indicates that the flexed position was an easy position for the index finger muscles (FDS and FDP), which assumes that coordinated precision grip force between the thumb and the index finger provides the force, making it difficult for subjects to coordinate the fine adjustment of FDS and FDP. Also, when the little finger was in the extended position, a minus error occurred that was larger than the other positions of the little finger. It is believed that it was difficult for subjects to finely coordinate the adjustment of FDS and FDP force as the FDS and FDP were extended. Therefore, subjects couldn't raise the following wave to the TW. Furthermore, when the little finger was performing joint movements, the flexor muscle contracted during a flexed movement. The flexor muscle contraction of the index finger influenced the coordinated precision grip force between the thumb and the index finger. In the little finger extension, the digital extensor muscle and the extensor digiti minimi muscle contracted. Like the flexion muscles (FDS and FDP), the digital extensor muscle was the common muscle during index and little finger extension. Hence, when pinching using the precision grip between the thumb and the index finger, the concentric contraction of radial digits and the efferent contraction of ulnar digits occurred simultaneously in the FDS and FDP. Furthermore, the concentric contraction of the little finger and the efferent contraction of the index finger occurred simultaneously in the digital extensor muscle. This suggests that the position of the little finger and the contraction of flexor and extensor muscles in the little finger, which had no involvement in precision grip between the thumb and the index finger, influenced power coordination. It may be that the little finger has a functional role in coordinating the grip force, even if the little finger isn't directly engaged in the grasping action. When the little finger joints' position were in a relaxed position, the contraction of FDS and FDP was neutral in the little finger, thus suggesting that keeping the muscle contraction of the little finger neutral was important for the fine-tuning of the precision grip force between the thumb and the index finger. The results point to the little finger having a functional role when coordinating grip force even if the little finger isn't directly engaged in the grasping action. In previous studies, the functional roles of the thumb and fingers have been found to take a direct part in prehension and manipulation. Taguchi (2002) suggested that each finger had a functional role in the rolling manipulation of a circular cylinder. This study indicates that the little finger position influences the coordinated precision grip force, which was not directly involved with the thumb and the index finger. Thus, to engage in prehension and manipulation of the digits, the thumb and digits may be related in all prehension and manipulation actions. It is necessary to consider not only the movement of every individual finger, and the movements of the whole hand which operate as a functional unit, but also where and how each finger influences another. The results of this study were obtained under limited conditions and parameters of the experiment. Thus, broader generalizations regarding the strategies of coordinated precision grip force from our study were difficult to make. Further research will investigate the coordinated precision grip force under many varying conditions.

Acknowledgements: The authors would like to give special thanks to all the participants and staff who facilitated in the data collection. This paper was supported by the Sapporo Medical University Graduate School of Health Sciences.

Declaration of interest: The authors report no conflicts of interest. The authors alone are responsible for the content and writing of paper.

\section{References}

Goto, M., \& Nakamura, M. (2003). The effect of wrist joint position for the pinch tasks. The Hokkaido Journal of Occupational Therapy. 20, 9-14 (in Japanese).

Johansson, RS., \& Westling, G. (1984). Roles of glabrous skin receptors and sensorimotor memory in automatic control of precision grip when lifting rougher or more slippery objects. Experimental Brain Research. 56, 550 564.

Kamakura, N., Omura, M., Ishii, H., Mitsuboshi, F., \& Miura Y. (1978). Positional patterns for prehension in normal hands. The Japanese Journal of Rehabilitation Medicine. 15, 65-82 (in Japanese).

Nakajima, R., Shibata, K., Yoneda, M., Inumaru, T., Nishimura, S., Chiba, K., \& Shimizu, J. (2012). Adjustment of upper-limb and finger motions for grasping a peg -Influences of obstacle positions on joint angles, trajectory and velocity-. Japanese Journal of Occupational Therapy Research. 14, 1-7 (in Japanese).

Nakamura, M., Sawada, Y., \& Tsubota, S. (1993). The Influence of Lord Force Perturbation on the control of precision grip during lifting tasks. Japanese Occupational Therapy Research. 12, 259-268 (in Japanese).

Napier, JR. (1956). The prehensile movements of the human hand. Journal of Bone and Joint Surgery B Nov., 38-B, 902-913.

Taguchi, H., Hase, K., \& Maeno, T. (2002). Analysis of the motion pattern and the learning mechanism for manip- 
ulating objects by human fingers. Transactions of the japan society of mechanical engineers, 68, 1647-1654 (in Japanese).

Yamashita, T., Kasai, T., Takahashi, M., Kurumatani, H., \& Murakami, T. (2005). Motor adaptation learning of repetitive precision grip task: motor behavioral and neurophysiological analysis. Japanese Journal of Clinical Neurophysiology. 33, 131-140 (in Japanese).
Zatsiorsky, VM., Gao, F., \& Latash, ML. (2003). Prehension synergies: Effects of object geometry and prescribed torques. Experimental Brain Research. 148, 77-87.

Zatsiorsky, VM., Gao, F., \& Latash, ML. (2003). Finger force vectors in multi-finger prehension. Journal of Biomechanics. 36, 1745-1749. 